\title{
Nicole Baumann
}

Nicole Baumann (1932-2017) a été pendant très longtemps un pilier de la Société de Biologie. Elle a fait partie à plusieurs reprises du Conseil et fréquenté assidûment les séances pendant des années. C'est donc une fidèle et une amie qui nous a quittés.

Médecin neurobiologiste, elle a contribué efficacement au développement de la neurochimie clinique en France, aux interfaces cliniques et biologiques dans le domaine des maladies neuro-métaboliques d'origine génétique de l'adulte et des maladies auto-immunes du nerf périphérique.

Dans les années 1970, Nicole Baumann crée le premier laboratoire de neurochimie, dans le service du professeur Paul Castaigne, à l'hôpital de la Pitié-Salpêtrière. Avec ses collaborateurs, elle a été l'une des premières à explorer des mutants neurologiques de la souris, conservés au laboratoire Jackson dans le Maine (États-Unis), en tant que modèles de maladies neurologiques. Elle s'est particulièrement intéressée aux mutants présentant un trouble du développement de la gaine de myéline (leucodystrophie), constituant si important pour la conduction normale de l'influx nerveux, qu'elle a caractérisé.

Nicole Baumann et ses collaborateurs ont également attiré l'attention sur la révélation tardive, chez l'adolescent et l'adulte, de maladies génétiques, habituellement considérées comme des maladies de l'enfant, impliquant la dégradation ou le transport de sphingolipides (neurolipidoses, leucodystrophies). Ils ont développé un laboratoire d'investigations diagnostiques biochimiques pour ces maladies neuro-métaboliques de l'adulte et, ensuite, contribué à développer une consultation pluridisciplinaire dans ce domaine avec le service de Génétique Clinique de la Pitié-Salpêtrière. Ils ont, enfin, mis en place un centre de référence pour les maladies lysosomales à expression neurologique.

Par ailleurs, avec sa collaboratrice Madeleine Gumpel, issue de l'Institut d'Embryologie Cellulaire et Moléculaire de Nogent-sur Marne, Nicole Baumann a développé des recherches sur la biologie des cellules gliales, partenaires des neurones, et sur les mécanismes de réparation du système nerveux par les techniques de transplantations cellulaires. Ces travaux ont été à l'origine de techniques expérimentales de thérapie cellulaire dans les maladies dys- et démyélinisantes. 